TRANSACTIONS OF THE

AMERICAN MATHEMATICAL SOCIETY

Volume 355, Number 9, Pages 3715-3726

S 0002-9947(03)03244-6

Article electronically published on May 7, 2003

\title{
PSEUDO-HOLOMORPHIC CURVES IN COMPLEX GRASSMANN MANIFOLDS
}

\author{
XIAOXIANG JIAO AND JIAGUI PENG
}

\begin{abstract}
It is proved that the Kähler angle of the pseudo-holomorphic sphere of constant curvature in complex Grassmannians is constant. At the same time we also prove several pinching theorems for the curvature and the Kähler angle of the pseudo-holomorphic spheres in complex Grassmannians with non-degenerate associated harmonic sequence.
\end{abstract}

\section{INTRODUCTION}

In this paper we study conformal minimal two-spheres in complex Grassmann manifolds by using the harmonic sequence. Given a harmonic map $\varphi$ of surfaces $M$ into the complex Grassmannian $\mathbf{G}_{k, n}$, by using the $\partial^{\prime}$-transform Chern and Wolfson ([3], [10]) obtained the following harmonic sequence associated to $\varphi$ :

$$
\varphi=\varphi_{0} \stackrel{\partial^{\prime}}{\longrightarrow} \varphi_{1} \stackrel{\partial^{\prime}}{\longrightarrow} \cdots \stackrel{\partial^{\prime}}{\longrightarrow} \varphi_{j} \stackrel{\partial^{\prime}}{\longrightarrow} \cdots
$$

where $\varphi_{j+1}=\partial^{\prime} \varphi_{j}, j=0,1, \cdots$, and $\varphi_{j}: M \rightarrow \mathbf{G}_{k_{j}, n}$ are harmonic maps, $k_{j}=$ $\operatorname{rank}\left(\varphi_{j}\right)$. If $\varphi_{j}$ is anti-holomorphic, then $k_{j+1}=0$. When $\varphi$ is holomorphic we call $\varphi_{j}$ a pseudo-holomorphic curve generated by $\varphi$. Such curves with the induced metrics from the associated complex Grassmann manifolds form a class of minimal immersions. When $k_{j}=k_{j+1}$ we say that $\varphi_{j}$ is non-degenerate. When $k_{j}=k_{j+1}$ for all $j$ we say that the harmonic sequence associated to the map $\varphi$ is non-degenerate.

When specialized to $\mathbf{G}_{1, n}=\mathbf{C} \mathbf{P}^{n-1}$, any pseudo-holomorphic curve is obtained from a holomorphic curve projected into $\mathbf{C P}^{n-1}$. Calabi ([2]) showed that any simply connected holomorphic curve in $\mathbf{C P}^{n-1}$ is completely determined, up to holomorphic isometries of $\mathbf{C P}^{n-1}$, by its induced metric. Calabi also showed that a simply connected holomorphic curve of constant curvature in $\mathbf{C} \mathbf{P}^{n-1}$ is the Veronese curve, up to unitary equivalence. For a pseudo-holomorphic curve in $\mathbf{C P}^{n-1}$, Bolton, Jensen, Rigoli and Woodward ([1]) showed that, up to a holomorphic isometry of $\mathbf{C P}^{n-1}$, the harmonic sequence determined by any linearly full conformal minimal immersion of constant curvature in $\mathbf{C} \mathbf{P}^{n-1}$ is the Veronese sequence, in which each map is a minimal immersion with constant curvature and constant Kähler angle.

Received by the editors September 6, 2002 and, in revised form, October 31, 2002.

2000 Mathematics Subject Classification. Primary 53C42, 53C55.

Key words and phrases. Gauss curvature, Kähler angle, harmonic sequence, pseudoholomorphic curve.

Supported by the National Natural Science Foundation of China (Grants No. 10001033, 10131020, 10071804) and the President Foundation of the Graduate School of the Chinese Academy of Sciences. 
It is well known that the rigidity fails for pseudo-holomorphic curves or holomorphic curves generalized to $\mathbf{G}_{k, n}$ ([5], [14]). For example, Chi and Zheng ([5]) classified the holomorphic curves of the Riemann sphere into $\mathbf{G}_{2,4}$ with the induced constant curvature 2 into two classes, up to unitary equivalence, in which none of the curves are congruent. Let $\varphi: \mathbf{S}^{2} \rightarrow \mathbf{G}_{k, n}$ be a pseudo-holomorphic curve in a complex Grassmannian $\mathbf{G}_{k, n}$. Problem: Is the Kähler angle $\theta(\varphi)$ of $\varphi$ constant when its Gauss curvature $K(\varphi)$ is constant? What are the relationships between the Kähler angle and the Gauss curvature of $\varphi$ and its ramification index? In this paper we will investigate these questions.

In the second and third sections of this paper we obtain some fundamental formulas for pseudo-holomorphic curves in complex Grassmann manifolds.

In the fourth section, by using these formulas we prove that the curvatures of pseudo-holomorphic curves are equal to $4 / N$ ( $N$ is a positive integer) if these curvatures are constant (this result was proved by Chi and Zheng in [5])(Theorem 4.1), and prove that Kähler angles of pseudo-holomorphic curves of constant curvature are constant (Theorem 4.2). In this section, we also give a harmonic sequence, in which each map is a minimal immersion with constant curvature and constant Kähler angle.

In the final section, we give some pinching theorems for pseudo-holomorphic curves with the associated non-degenerate harmonic sequence for curvatures and Kähler angles (Theorems 5.2, 5.6 and 5.7). At the same time we also show that the Kähler angle of a pseudo-holomorphic curve is independent of its ramification index under the assumption of Theorem 5.2.

\section{Minimal Immersions and Harmonic Sequences}

Let $U(n)$ be the unitary group. Let $M$ be a simply connected domain in the unit sphere $\mathbf{S}^{2}$ and let $(z, \bar{z})$ be a complex coordinate on $M$. We take the metric $d s_{M}^{2}=d z d \bar{z}$ on $M$. Denote

$$
\partial=\frac{\partial}{\partial z}, \quad \bar{\partial}=\frac{\partial}{\partial \bar{z}}, \quad A_{z}=\frac{1}{2} s^{-1} \partial s, \quad A_{\bar{z}}=\frac{1}{2} s^{-1} \bar{\partial} s .
$$

Let $s: M \rightarrow U(n)$ be a smooth map; then $s$ is a harmonic map if and only if it satisfies the following equation $([9])$ :

$$
\bar{\partial} A_{z}=\left[A_{z}, A_{\bar{z}}\right] .
$$

If $s: \mathbf{S}^{2} \rightarrow U(n)$ is a harmonic map, then $s$ is a conformal map; so $s$ is a minimal immersion. Let $\omega=g^{-1} d g$ be a Maurer-Cartan form on $U(n)$, and let $d s_{U(n)}^{2}=$ $\frac{1}{8} \operatorname{tr} \omega \omega^{*}$ be the metric on $U(n)$. Then the metric induced by $s$ on $\mathbf{S}^{2}$ is given by

$$
d s^{2}=-\operatorname{tr} A_{z} A_{\bar{z}} d z d \bar{z} .
$$

Let $\mathbf{G}_{k, n}$ be the complex Grassmann manifold consisting of all complex $k$-dimensional subspaces in $\mathbf{C}^{n}$. Here we consider $\mathbf{G}_{k, n}$ as the set of Hermitian orthogonal projections onto a $k$-dimensional subspace in $\mathbf{C}^{n}$, i.e., $\mathbf{G}_{k, n}=\{\varphi$ is the Hermitian orthogonal projection onto a $k$-dimensional subspace in $\left.\mathbf{C}^{n}\right\}$. Then $\varphi: \mathbf{S}^{2} \rightarrow \mathbf{G}_{k, n}$ is a Hermitian orthogonal projection onto a $k$-dimensional subbundle $\eta \subset \mathbf{S}^{2} \times \mathbf{C}^{n}$, and $s=\varphi-\varphi^{\perp}$ is a map from $\mathbf{S}^{2}$ into $U(n)$. It is well known that $\varphi$ is harmonic if and only if $s$ is harmonic. If $\varphi^{\perp} \bar{\partial} \varphi=0$ or $\varphi^{\perp} \partial \varphi=0$, we call $\varphi$ a holomorphic curve or an anti-holomorphic curve in $\mathbf{G}_{k, n}$. 
Using $\varphi$, the harmonic sequences (see [3], [10]) are given by

$$
\begin{gathered}
\varphi=\varphi_{0} \stackrel{\partial^{\prime}}{\longrightarrow} \varphi_{1} \stackrel{\partial^{\prime}}{\longrightarrow} \cdots \stackrel{\partial^{\prime}}{\longrightarrow} \varphi_{\alpha} \stackrel{\partial^{\prime}}{\longrightarrow} \cdots, \\
\varphi=\varphi_{0} \stackrel{\partial^{\prime \prime}}{\longrightarrow} \varphi_{-1} \stackrel{\partial^{\prime \prime}}{\longrightarrow} \cdots \stackrel{\partial^{\prime \prime}}{\longrightarrow} \varphi_{-\alpha} \stackrel{\partial^{\prime \prime}}{\longrightarrow} \cdots,
\end{gathered}
$$

where $\varphi_{\alpha}: \mathbf{S}^{2} \times \mathbf{C}^{n} \rightarrow \operatorname{Im}\left(\varphi_{\alpha-1}^{\perp} \partial \varphi_{\alpha-1}\right)$ and $\varphi_{-\alpha}: \mathbf{S}^{2} \times \mathbf{C}^{n} \rightarrow \operatorname{Im}\left(\varphi_{-\alpha+1}^{\perp} \bar{\partial} \varphi_{-\alpha+1}\right)$ are Hermitian orthogonal projections, $\alpha=1,2, \cdots$.

Proposition 2.1 ([7]). For (3) and (4), we have

$$
\varphi_{\alpha} \partial \varphi_{\alpha}=-\varphi_{\alpha-1}^{\perp} \partial \varphi_{\alpha-1}, \quad \varphi_{\alpha}^{\perp} \bar{\partial} \varphi_{\alpha}=-\varphi_{\alpha-1} \bar{\partial} \varphi_{\alpha-1},
$$

where $\alpha= \pm 1, \pm 2, \cdots$.

If $\varphi_{0}$ is a holomorphic curve in (3) or an anti-holomorphic curve in (4), then elements in (3) or (4) are finite and are mutually orthogonal. If there exists a holomorphic curve $\varphi_{0}$ in $\mathbf{G}_{k, n}$ such that $\varphi$ is an element in the harmonic sequence (3), i.e., $\varphi=\varphi_{\alpha}: \mathbf{S}^{2} \rightarrow \mathbf{G}_{k_{\alpha}, n}$ belongs to the harmonic sequence

$$
0 \stackrel{\partial^{\prime}}{\longrightarrow} \varphi_{0} \stackrel{\partial^{\prime}}{\longrightarrow} \varphi_{1} \stackrel{\partial^{\prime}}{\longrightarrow} \cdots \stackrel{\partial^{\prime}}{\longrightarrow} \varphi=\varphi_{\alpha} \stackrel{\partial^{\prime}}{\longrightarrow} \cdots \stackrel{\partial^{\prime}}{\longrightarrow} \varphi_{\alpha_{0}} \stackrel{\partial^{\prime}}{\longrightarrow} 0,
$$

then we call $\varphi$ a pseudo-holomorphic curve in complex Grassmann manifolds, and $\alpha_{0}$ is called the length of the harmonic sequence (5).

Now we assume that $\varphi=\varphi_{\alpha}: \mathbf{S}^{2} \rightarrow \mathbf{G}_{k_{\alpha}, n}$ is a pseudo-holomorphic curve. Then we may choose the local unitary frame $e_{1}, e_{2}, \cdots, e_{n}$ on $\mathbf{S}^{2} \times \mathbf{C}^{n}$ such that $e_{k_{\alpha-1}+1}, \cdots, e_{k_{\alpha}} \operatorname{span} \operatorname{Im}\left(\varphi_{\alpha-1}^{\perp} \partial \varphi_{\alpha-1}\right)$, where $k_{\alpha}=\operatorname{rank}\left(\varphi_{\alpha-1}^{\perp} \partial \varphi_{\alpha-1}\right), \alpha=$ $1,2, \cdots, k_{0}=\operatorname{rank}\left(\varphi_{0}\right)$.

Let $W_{\alpha}=\left(e_{k_{\alpha-1}+1}, e_{k_{\alpha}}, \cdots, e_{k_{\alpha}}\right)$ be an $\left(n \times k_{\alpha}\right)$-matrix. Then we have

$$
\begin{gathered}
\varphi_{\alpha}=W_{\alpha} W_{\alpha}^{*}, \\
W_{\alpha}^{*} W_{\alpha}=I_{k_{\alpha} \times k_{\alpha}}, \quad W_{\alpha}^{*} W_{\alpha+1}=0, \quad W_{\alpha}^{*} W_{\alpha-1}=0 .
\end{gathered}
$$

By (7) and a straightforward computation we obtain

$$
\left\{\begin{array}{l}
\partial W_{\alpha}=W_{\alpha+1} \Omega_{\alpha}+W_{\alpha} \Psi_{\alpha} \\
\bar{\partial} W_{\alpha}=-W_{\alpha-1} \Omega_{\alpha-1}^{*}-W_{\alpha} \Psi_{\alpha}^{*}
\end{array}\right.
$$

where $\Omega_{\alpha}$ is a $\left(k_{\alpha+1} \times k_{\alpha}\right)$-matrix and $\Psi_{\alpha}$ is a $\left(k_{\alpha} \times k_{\alpha}\right)$-matrix, $\alpha=0,1,2, \cdots$.

It is well known that $\Omega_{\alpha}=0$ or $\Omega_{\alpha-1}=0$ in (8) if and only if $\varphi_{\alpha}$ is antiholomorphic or holomorphic. It is very evident that integrability conditions for (8) are

$$
\begin{gathered}
\bar{\partial} \Omega_{\alpha}=\Psi_{\alpha+1}^{*} \Omega_{\alpha}-\Omega_{\alpha} \Psi_{\alpha}^{*} \\
\bar{\partial} \Psi_{\alpha}+\partial \Psi_{\alpha}^{*}=\Omega_{\alpha}^{*} \Omega_{\alpha}+\Psi_{\alpha}^{*} \Psi_{\alpha}-\Omega_{\alpha-1} \Omega_{\alpha-1}^{*}-\Psi_{\alpha} \Psi_{\alpha}^{*} .
\end{gathered}
$$

By (8), $A_{z}^{(\alpha)}$ and $A_{\bar{z}}^{(\alpha)}$ for $\varphi_{\alpha}$ are given by

$$
\begin{gathered}
A_{z}^{(\alpha)}=-W_{\alpha} \Omega_{\alpha-1} W_{\alpha-1}^{*}-W_{\alpha+1} \Omega_{\alpha} W_{\alpha}^{*}, \\
A_{\bar{z}}^{(\alpha)}=W_{\alpha} \Omega_{\alpha}^{*} W_{\alpha+1}^{*}+W_{\alpha-1} \Omega_{\alpha}^{*} W_{\alpha}^{*} .
\end{gathered}
$$

It can easily be checked that (9) is equivalent to (1). An immediate consequence of $(8)$ is 
Proposition 2.2. Let $\varphi=\varphi_{\alpha}: \mathbf{S}^{2} \rightarrow \mathbf{G}_{k_{\alpha}, n}$ be a pseudo-holomorphic curve, with $\Omega_{\alpha}$ and $\Psi_{\alpha}$ determined by equations (8). Then $\Omega_{\alpha}$ and $\Psi_{\alpha}$ satisfy equations (9) and (10).

Let $\varphi^{(\alpha)}=\varphi_{0} \oplus \cdots \oplus \varphi_{\alpha}$ for $(5)$ and $k_{(\alpha)}=k_{0}+\cdots+k_{\alpha}$. Then by Proposition 2.1 we have

$$
\partial \varphi^{(\alpha)}=\varphi_{\alpha}^{\perp} \partial \varphi_{\alpha}
$$

Hence $\varphi^{(\alpha)}: \mathbf{S}^{2} \rightarrow \mathbf{G}_{k_{(\alpha)}, n}$ is a holomorphic map, and the harmonic map sequence (5) becomes

$$
0 \stackrel{\partial^{\prime}}{\longrightarrow} \varphi^{(\alpha)} \stackrel{\partial^{\prime}}{\longrightarrow} \varphi_{\alpha+1} \stackrel{\partial^{\prime}}{\longrightarrow} \cdots \stackrel{\partial^{\prime}}{\longrightarrow} \varphi_{\alpha_{0}} \stackrel{\partial^{\prime}}{\longrightarrow} 0 .
$$

If $k_{\alpha}=k_{\alpha+1}$, i.e., $\operatorname{rank}\left(\varphi_{\alpha}\right)=\operatorname{rank}\left(\varphi_{\alpha+1}\right)$, then $\varphi_{\alpha}$ is called non-degenerate. If $\varphi_{\alpha}$ is non-degenerate for $\alpha=0,1, \cdots, \alpha_{0}-1$ in (5), i.e., $k_{0}=k_{1}=\cdots=k_{\alpha_{0}}$, then the harmonic sequence (5) is called the non-degenerate harmonic sequence associated to the harmonic map $\varphi=\varphi_{\alpha}$. Now we assume that $\varphi_{\alpha}$ is non-degenerate; then $\operatorname{det}\left(\Omega_{\alpha}\right)$ is a well-defined invariant on $\mathbf{S}^{2}$ and has only isolated zeros. Let

$$
l_{\alpha}=\operatorname{tr}\left(\Omega_{\alpha} \Omega_{\alpha}^{*}\right) .
$$

Then

$$
l_{\alpha}=\operatorname{tr}\left(\varphi_{\alpha}^{\perp} \partial \varphi_{\alpha} \bar{\partial} \varphi_{\alpha}\right)=\operatorname{tr}\left(\partial \varphi^{(\alpha)} \bar{\partial} \varphi^{(\alpha)}\right), \quad l_{\alpha-1}+l_{\alpha}=-\operatorname{tr}\left(A_{z}^{(\alpha)} A_{\bar{z}}^{(\alpha)}\right),
$$

and we have

Proposition 2.3. If $\varphi=\varphi_{\alpha}: \mathbf{S}^{2} \rightarrow \mathbf{G}_{k_{\alpha}, n}$ is a non-degenerate pseudo-holomorphic curve, then

$$
2 \partial \bar{\partial} \log \left|\operatorname{det}\left(\Omega_{\alpha}\right)\right|=l_{\alpha-1}-2 l_{\alpha}+l_{\alpha+1} .
$$

Proof. By (9) and the rule of differentiating a determinant, we get

$$
\begin{gathered}
\bar{\partial} \log \operatorname{det}\left(\Omega_{\alpha}\right)=\operatorname{tr}\left(\Omega_{\alpha}^{-1} \bar{\partial} \Omega_{\alpha}\right)=\operatorname{tr} \Psi_{\alpha+1}^{*}-\operatorname{tr} \Psi_{\alpha}^{*}, \\
\partial \log \operatorname{det}\left(\Omega_{\alpha}^{*}\right)=\operatorname{tr}\left(\left(\Omega_{\alpha}^{*}\right)^{-1} \partial \Omega_{\alpha}^{*}\right)=\operatorname{tr} \Psi_{\alpha+1}-\operatorname{tr} \Psi_{\alpha} .
\end{gathered}
$$

It is not difficult to obtain (16) by (10).

Remark. If $\varphi_{\alpha}$ is non-degenerate for all $\alpha$ in (5), then

$$
2 \partial \bar{\partial} \log \left|\operatorname{det}\left(\Omega_{\alpha}\right)\right|=l_{\alpha-1}-2 l_{\alpha}+l_{\alpha+1}
$$

for $\alpha=0,1, \cdots, \alpha_{0}-1$, where $l_{-1}=l_{\alpha_{0}}=0$. When $k_{\alpha}=1$ for all $\alpha$, then $l_{\alpha}=\left|\operatorname{det}\left(\Omega_{\alpha}\right)\right|^{2}$, and (17) is just the unintegrated Plücker formulae for $l_{\alpha}$ derived by Bolton, Jensen, Rigoli and Woodward in [1].

\section{Kähler Angles and Gauss Curvatures}

If $\varphi: M \rightarrow \mathbf{G}_{k, n}$ is a conformal immersion of a Riemann surface $M$, we define the Kähler angle of $\varphi$ to be the function $\theta: M \rightarrow[0, \pi]$ given in terms of a complex coordinate $z$ on $M$ by

$$
\tan \frac{\theta(p)}{2}=\frac{|d \varphi(\partial / \partial \bar{z})|}{|d \varphi(\partial / \partial z)|}, \quad p \in M
$$

It is clear that $\theta$ is globally defined and is smooth at $p$ unless $\theta(p)=0$ or $\pi$. Let $z=x+\sqrt{-1} y$, and let $J$ denote the complex structure on $\mathbf{G}_{k, n}$; then $\theta$ is the angle between $J d \varphi(\partial / \partial x)$ and $d \varphi(\partial / \partial y)$. The importance of the Kähler angle in 
the theory of minimal immersions of surfaces into Kähler manifolds was pointed out by Chern and Wolfson [4. Indeed, $\varphi$ is holomorphic if and only if $\theta(p)=0$ for all $p \in M$, while $\varphi$ is anti-holomorphic if and only if $\theta(p)=\pi$ for all $p \in M$.

Now suppose that $\varphi: \mathbf{S}^{2} \rightarrow \mathbf{G}_{k, n}$ is a conformal minimal immersion in the harmonic sequence (5). Then each $\varphi_{\alpha}: \mathbf{S}^{2} \rightarrow \mathbf{G}_{k_{\alpha}, n}$ is a conformal minimal immersion. So there exists a finite set $X_{\alpha}$ (see [1]) such that the Kähler angle

$$
\theta_{\alpha}: \mathbf{S}^{2} \backslash X_{\alpha} \rightarrow[0, \pi]
$$

is well defined, and is smooth on $\mathbf{S}^{2} \backslash X_{\alpha}$.

Let $t_{\alpha}=\left(\tan \frac{\theta_{\alpha}}{2}\right)^{2}$. Then, in terms of a local complex coordinate $z$,

$$
t_{\alpha}=\frac{\left|d \varphi_{\alpha}(\partial / \partial \bar{z})\right|^{2}}{\left|d \varphi_{\alpha}(\partial / \partial z)\right|^{2}}=\frac{l_{\alpha-1}}{l_{\alpha}} .
$$

Let $d s_{\alpha}^{2}$ and $d s_{(\alpha)}^{2}$ be the metrics on $\mathbf{S}^{2} \backslash X_{\alpha}$ induced by $\varphi_{\alpha}$ and $\varphi^{(\alpha)}$ respectively. Then by (11), (12) and (13) we have

$$
d s_{\alpha}^{2}=\left(l_{\alpha-1}+l_{\alpha}\right) d z d \bar{z}, \quad d s_{(\alpha)}^{2}=l_{\alpha} d z d \bar{z} .
$$

The Laplacians $\triangle_{\alpha}$ and $\triangle_{(\alpha)}$ for $d s_{\alpha}^{2}$ and $d s_{(\alpha)}^{2}$ are given by

$$
\triangle_{\alpha}=\frac{4}{l_{\alpha-1}+l_{\alpha}} \partial \bar{\partial}, \quad \triangle_{(\alpha)}=\frac{4}{l_{\alpha}} \partial \bar{\partial}
$$

and the curvatures $K_{\alpha}, K_{(\alpha)}$ of $\varphi_{\alpha}$ and $\varphi^{(\alpha)}$ by

$$
K_{\alpha}=-\frac{2}{l_{\alpha-1}+l_{\alpha}} \partial \bar{\partial} \log \left(l_{\alpha-1}+l_{\alpha}\right), \quad K_{(\alpha)}=-\frac{2}{l_{\alpha}} \partial \bar{\partial} \log l_{\alpha},
$$

the area forms $d v_{\alpha}$ and $d v_{(\alpha)}$ by

$$
d v_{\alpha}=\left(l_{\alpha-1}+l_{\alpha}\right) \frac{d \bar{z} \wedge d z}{2 \sqrt{-1}}, \quad d v_{(\alpha)}=l_{\alpha} \frac{d \bar{z} \wedge d z}{2 \sqrt{-1}} .
$$

Choose holomorphic sections $f_{1}, \cdots, f_{k_{(\alpha)}}$ in $\Gamma\left(\mathbf{S}^{2} \times \mathbf{C}^{n}\right)$ so that they $\operatorname{span} \operatorname{Im}\left(\varphi^{(\alpha)}\right)$ and

$$
f_{1} \wedge \cdots \wedge f_{k_{(\alpha)}}: \mathbf{S}^{2} \rightarrow \mathbf{C}^{\left(\begin{array}{c}
n \\
k_{(\alpha)}
\end{array}\right)}
$$

is a nowhere zero holomorphic curve.

Let $F^{(\alpha)}=f_{1} \wedge \cdots \wedge f_{k_{(\alpha)}}$. Now consider the Plücker embedding (see [12, 13])

$$
\left[F^{(\alpha)}\right]: \mathbf{S}^{2} \rightarrow \mathbf{C P}^{\left(\begin{array}{c}
n \\
k_{(\alpha)}
\end{array}\right)-1},
$$

which is a holomorphic isometry, and

$$
\left[F^{(\alpha)}\right]^{*} d s^{2} \mathbf{C P}_{\left(\begin{array}{c}
n \\
k(\alpha)
\end{array}\right)-1}=l_{\alpha} d z d \bar{z} .
$$

By [1, we have

$$
\partial \bar{\partial} \log \left|F^{(\alpha)}\right|^{2}=l_{\alpha}
$$

and the degree $\delta_{\alpha}$ of $F^{(\alpha)}$ is given by

$$
\delta_{\alpha}=\frac{1}{2 \pi \sqrt{-1}} \int_{\mathbf{S}^{2}} \partial \bar{\partial} \log \left|F^{(\alpha)}\right|^{2} d \bar{z} \wedge d z=\frac{1}{2 \pi \sqrt{-1}} \int_{\mathbf{S}^{2}} l_{\alpha} d \bar{z} \wedge d z,
$$


which is equal to the degree of the polynomial function $F^{(\alpha)}$ in $z$. We call $\delta_{\alpha}$ the degree of the holomorphic curve $\varphi^{(\alpha)}$. Thus from (17) and (27) we get

Proposition 3.1. If $\varphi=\varphi_{\alpha}: \mathbf{S}^{2} \rightarrow \mathbf{G}_{k_{\alpha}, n}$ is a non-degenerate pseudo-holomorphic curve, then

$$
-\sharp_{\alpha}=\delta_{\alpha-1}-2 \delta_{\alpha}+\delta_{\alpha+1},
$$

where $\sharp_{\alpha}=-\frac{1}{\pi \sqrt{-1}} \int_{\mathbf{S}^{2}} \partial \bar{\partial} \log \left|\operatorname{det} \Omega_{\alpha}\right| d \bar{z} \wedge d z$ is the number of singular points of $\Omega_{\alpha}$, i.e., the number of zeros of $\operatorname{det} \Omega_{\alpha}$.

Remark. If $\varphi_{\alpha}$ is non-degenerate for $\alpha=0,1, \cdots, \alpha_{0}-1$, then $-\sharp_{\alpha}=\delta_{\alpha-1}-2 \delta_{\alpha}+$ $\delta_{\alpha+1}$ for all $\alpha$, and $\delta_{-1}=\delta_{\alpha_{0}}=0$; in particular, when $k_{0}=\cdots=k_{\alpha_{0}}=1,(28)$ is the global Plücker formula (see [6]).

Let $d s^{2}=\left|\operatorname{det} \Omega_{\alpha}\right|^{2} d z d \bar{z}=\psi_{\alpha} \bar{\psi}_{\alpha}$, where $\psi_{\alpha}$ is a type $(1,0)$ analytic 1-form. Then $d s^{2}=\psi_{\alpha} \oplus \bar{\psi}_{\alpha}$ is a singular Hermitian metric. Let $D_{S}=\sum_{p \in \mathbf{S}^{2}} \operatorname{ord}_{p}\left(\psi_{\alpha}\right) p$ be the singular divisor of $\left(\mathbf{S}^{2}, d s^{2}\right)$, i.e., the zero divisor of $\psi_{\alpha}$. By the Gauss-BonnetChern theorem we have

$$
\sharp_{\alpha}=\tau_{\alpha}+2,
$$

where $\tau_{\alpha}=\operatorname{deg} D_{S}$.

We say that $\tau_{\alpha}$ is the ramification index of $\varphi_{\alpha}$. Evidently, $\tau_{\alpha}$ is a non-negative integer. If $\tau_{\alpha}=0, \varphi_{\alpha}$ is called unramified by Bolton et al. ([1]).

Let (5) be the non-degenerate harmonic sequence; if $\tau_{\alpha}=0$ for $\alpha=0,1, \cdots, \alpha_{0}-$ 1 , the harmonic sequence (5) is called totally unramified. Let $\varphi=\varphi_{\alpha}: \mathbf{S}^{2} \rightarrow$ $\mathbf{G}_{k_{\alpha}, n}$ be the pseudo-holomorphic conformal immersion with the non-degenerate associated harmonic sequence (5); we say that $\varphi$ is a totally unramified pseudoholomorphic conformal immersion if $\varphi_{0}, \cdots, \varphi_{\alpha_{0}}$ is totally unramified.

If $\varphi_{\alpha}: \mathbf{S}^{2} \rightarrow \mathbf{G}_{k_{\alpha}, n}$ is a conformal minimal immersion with constant Kähler angle, then we have

$$
t_{\alpha}=\frac{\delta_{\alpha-1}}{\delta_{\alpha}}
$$

and from (19) and (22) it follows that

$$
K_{\alpha}=-\frac{2}{l_{\alpha-1}+l_{\alpha}} \partial \bar{\partial} \log l_{\alpha-1}=-\frac{2}{l_{\alpha-1}+l_{\alpha}} \partial \bar{\partial} \log l_{\alpha} .
$$

\section{Conformal Minimal Immersions with Constant Curvatures}

It is well known that any complex submanifold of a (simply-connected, complete) space of constant holomorphic curvature is completely determined, up to holomorphic isometries of the ambient space, by its induced metric (see [2], [8]). The Veronese sequence is the harmonic sequence

$$
0 \stackrel{\partial^{\prime}}{\longrightarrow} \varphi_{0} \stackrel{\partial^{\prime}}{\longrightarrow} \cdots \stackrel{\partial^{\prime}}{\longrightarrow} \varphi_{n} \stackrel{\partial^{\prime}}{\longrightarrow} 0,
$$

where $n=\operatorname{deg}\left(\varphi_{0}\right)$, and each $\varphi_{\alpha}=\left[g_{\alpha, 0}, \cdots, g_{\alpha, n}\right]: \mathbf{S}^{2} \rightarrow \mathbf{C P}^{n}$ is given by

$$
g_{\alpha, j}=\frac{\alpha !}{(1+z \bar{z})^{\alpha}} \sqrt{\left(\begin{array}{c}
n \\
j
\end{array}\right)} z^{j-\alpha} \sum_{k}(-1)^{k}\left(\begin{array}{c}
j \\
\alpha-k
\end{array}\right)\left(\begin{array}{c}
n-j \\
k
\end{array}\right)(z \bar{z})^{k}, \quad \alpha, j=0,1, \ldots, n .
$$


Each map $\varphi_{\alpha}$ in the Veronese sequence (31) is a conformal minimal immersion with constant curvature

$$
K\left(\varphi_{\alpha}\right)=\frac{4}{n+2 \alpha(n-\alpha)}
$$

and constant Kähler angle $\theta_{\alpha}$ given by

$$
\left(\tan \frac{\theta_{\alpha}}{2}\right)^{2}=\frac{\alpha(n-\alpha+1)}{(\alpha+1)(n-\alpha)}
$$

Bolton, Jensen, Rigoli and Woodward ([1]) showed that, up to a holomorphic isometry of $\mathbf{C P}^{n}$, the harmonic sequence determined by $\varphi: \mathbf{S}^{2} \rightarrow \mathbf{C P}^{n}$, which is a linearly full conformal minimal immersion of constant curvature, is the Veronese sequence. It is very complicated for pseudo-holomorphic curves in complex Grassmann manifolds; for example, rigidity fails, but we still believe that there are some good geometric properties. In this section we discuss pseudo-holomorphic curves of constant curvature in complex Grassmann manifolds, and Kähler angles.

Let $\varphi_{\alpha}: \mathbf{S}^{2} \rightarrow \mathbf{G}_{k_{\alpha}, n}$ be a pseudo-holomorphic curve with constant curvature. Then we know that

$$
\left[F^{(\alpha-1)}\right]: \mathbf{S}^{2} \rightarrow \mathbf{C P}^{\left(\begin{array}{c}
n \\
k_{(\alpha-1)}
\end{array}\right)-1}, \quad\left[F^{(\alpha)}\right]: \mathbf{S}^{2} \rightarrow \mathbf{C P}^{\left(\begin{array}{c}
n \\
k_{(\alpha)}
\end{array}\right)-1}
$$

are two holomorphic curves with degrees $\delta_{\alpha-1}$ and $\delta_{\alpha}$ respectively. Consider the tensor product of $\left[F^{(\alpha-1)}\right]$ and $\left[F^{(\alpha)}\right]$,

$$
T^{(\alpha)}=F^{(\alpha-1)} \otimes F^{(\alpha)} .
$$

Then

$$
\left[T^{(\alpha)}\right]: \mathbf{S}^{2} \rightarrow \mathbf{C P}^{\left(\begin{array}{c}
n \\
k_{(\alpha-1)}
\end{array}\right)\left(\begin{array}{c}
n \\
k_{(\alpha-1)}
\end{array}\right)-1}
$$

is a well-defined holomorphic curve, and from (25) the metric induced by $\left[T^{(\alpha)}\right]$ is given by

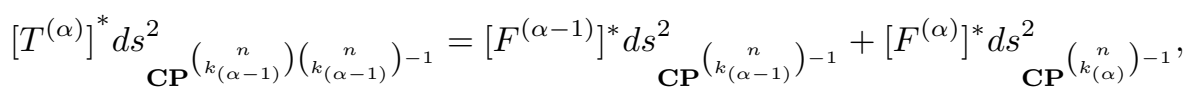

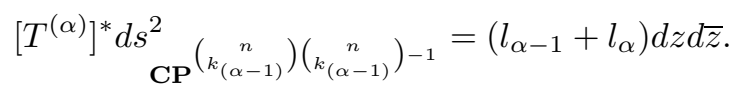

Hence the curvature $K_{\alpha}$ of $\varphi_{\alpha}$ is equal to the curvature of $\left[T^{(\alpha)}\right]$. From [1] an immediate consequence is

Theorem 4.1. If $\varphi: \mathbf{S}^{2} \rightarrow \mathbf{G}_{k, n}$ is a pseudo-holomorphic curve with constant curvature $K(\varphi)$, then $K(\varphi)=4 / N$, where $N$ is a positive integer.

This theorem was proved by Chi and Zheng ([5]) by the method of the moving frame. In the following we will prove

Theorem 4.2. If $\varphi_{\alpha}: \mathbf{S}^{2} \rightarrow \mathbf{G}_{k_{\alpha}, n}$ is a pseudo-holomorphic curve with constant curvature $K_{\alpha}$, then the Kähler angle $\theta_{\alpha}$ of $\varphi_{\alpha}$ is constant.

Proof. From (22) we have

$$
K_{\alpha}\left(l_{\alpha-1}+l_{\alpha}\right)=-2 \partial \bar{\partial} \log \left(l_{\alpha-1}+l_{\alpha}\right) .
$$


When $K_{\alpha}$ is constant, from (22), (23), (27) and the Gauss-Bonnet theorem it follows that

$$
K_{\alpha}=\frac{4}{\delta_{\alpha-1}+\delta_{\alpha}}
$$

Hence from (22) we obtain

$$
-\frac{2}{l_{\alpha-1}+l_{\alpha}} \partial \bar{\partial} \log \left(l_{\alpha-1}+l_{\alpha}\right)=\frac{4}{\delta_{\alpha-1}+\delta_{\alpha}} .
$$

Choose a complex coordinate $z$ on $\mathbf{S}^{2} \backslash\{p t\}$ so that

$$
l_{\alpha-1}+l_{\alpha}=\frac{\delta_{\alpha-1}+\delta_{\alpha}}{(1+z \bar{z})^{2}}
$$

From (38), (39) and (26) we obtain

$$
\partial \bar{\partial} \log \frac{\left|F^{(\alpha-1)}\right|^{2}\left|F^{(\alpha)}\right|^{2}}{(1+z \bar{z})^{\delta_{\alpha-1}+\delta_{\alpha}}}=0 .
$$

Since we can choose holomorphic sections $f_{1}, \cdots, f_{k_{(\alpha)}}$ in $\Gamma\left(\mathbf{S}^{2} \times \mathbf{C}^{n}\right)$ such that the maps $F^{(\alpha-1)}$ and $F^{(\alpha)}$ are polynomial functions on $\mathbf{C}$ of degrees $\delta_{\alpha-1}$ and $\delta_{\alpha}$ respectively, it folows that $\frac{\left|F^{(\alpha-1)}\right|^{2}\left|F^{(\alpha)}\right|^{2}}{(1+z \bar{z})^{\delta_{\alpha-1}+\delta_{\alpha}}}$ is globally defined on $\mathbf{C}$ and has a non-zero constant limit $c$, as $z \rightarrow \infty$. So from (40) we get

$$
\frac{\left|F^{(\alpha-1)}\right|^{2}\left|F^{(\alpha)}\right|^{2}}{(1+z \bar{z})^{\delta_{\alpha-1}+\delta_{\alpha}}}=c
$$

Then we have

$$
\left|F^{(\alpha-1)}\right|^{2}=c_{\alpha-1}(1+z \bar{z})^{\delta_{\alpha-1}}, \quad\left|F^{(\alpha)}\right|^{2}=c_{\alpha}(1+z \bar{z})^{\delta_{\alpha}},
$$

where $c_{\alpha-1}$ and $c_{\alpha}$ are constants.

Hence, $l_{\alpha-1}=\frac{\delta_{\alpha-1}}{(1+z \bar{z})^{2}}$ and $l_{\alpha}=\frac{\delta_{\alpha}}{(1+z \bar{z})^{2}}$, namely, $\varphi_{\alpha}$ is of constant curvature and constant Kähler angle.

From (19) and (22) we know that if $\varphi_{\alpha}: \mathbf{S}^{2} \rightarrow \mathbf{G}_{k_{\alpha}, n}$ is a pseudo-holomorphic curve with constant Kähler angle $\theta_{\alpha}$, then $K_{\alpha}=\frac{1}{1+t_{\alpha}} K_{(\alpha)}$.

Remark. We do not need to assume that $\varphi_{\alpha}: \mathbf{S}^{2} \rightarrow \mathbf{G}_{k_{\alpha}, n}$ is non-degenerate in Theorem 4.2.

To conclude this section, we give an example. This example is a harmonic sequence, in which the Gauss curvature and the Kähler angle of each element are constant.

Let $f_{0}(z)=\left(1,0, \sqrt{2} z, 0, z^{2}\right)$ and $g_{0}(z)=(0,1,0, z, 0)$; then

$$
\varphi_{0}=\frac{1}{(1+z \bar{z})^{2}}\left(\begin{array}{ccccc}
1 & 0 & \sqrt{2} z & 0 & z^{2} \\
0 & 1+z \bar{z} & 0 & z(1+z \bar{z}) & 0 \\
\sqrt{2} \bar{z} & 0 & 2 z \bar{z} & 0 & \sqrt{2} z^{2} \bar{z} \\
0 & \bar{z}(1+z \bar{z}) & 0 & z \bar{z}(1+z \bar{z}) & 0 \\
\bar{z}^{2} & 0 & \sqrt{2} z \bar{z}^{2} & 0 & z^{2} \bar{z}^{2}
\end{array}\right): \mathbf{S}^{2} \rightarrow \mathbf{G}_{2,5}
$$

determined by $f_{0}(z)$ and $g_{0}(z)$ is a holomorphic map. 
An immediate computation shows that

$$
\begin{gathered}
f_{1}(z, \bar{z})=\varphi_{0}^{\perp}\left(\partial f_{0}(z)\right)=\left(-\frac{2 \bar{z}}{1+z \bar{z}}, 0, \frac{\sqrt{2}(1-z \bar{z})}{1+z \bar{z}}, 0, \frac{2 z}{1+z \bar{z}}\right), \\
g_{1}(z, \bar{z})=\varphi_{0}^{\perp}\left(\partial g_{0}(z)\right)=\left(0,-\frac{\bar{z}}{1+z \bar{z}}, 0, \frac{1}{1+z \bar{z}}, 0\right),
\end{gathered}
$$

and $\varphi_{1}(z, \bar{z})$ determined by $f_{1}$ and $g_{1}$ is given by

$$
\varphi_{1}=\frac{1}{(1+z \bar{z})^{2}}\left(\begin{array}{ccccc}
2 z \bar{z} & 0 & \sqrt{2} z(z \bar{z}-1) & 0 & -2 z^{2} \\
0 & z \bar{z}(1+z \bar{z}) & 0 & -z(1+z \bar{z}) & 0 \\
\sqrt{2} \bar{z}(z \bar{z}-1) & 0 & (z \bar{z}-1)^{2} & 0 & \sqrt{2} z(z \bar{z}-1) \\
0 & -\bar{z}(1+z \bar{z}) & 0 & 1+z \bar{z} & 0 \\
-2 \bar{z}^{2} & 0 & \sqrt{2} \bar{z}(z \bar{z}-1) & 0 & 2 z \bar{z}
\end{array}\right) \text {, }
$$

which is obviously a pseudo-holomorphic curve into $\mathbf{G}_{2,5}$. Similarly, we have

$$
\begin{gathered}
f_{2}=\varphi_{1}^{\perp}\left(\partial f_{1}\right)=\left(\frac{2 \bar{z}^{2}}{(1+z \bar{z})^{2}}, 0,-\frac{2 \sqrt{2} \bar{z}}{(1+z \bar{z})^{2}}, 0, \frac{2}{(1+z \bar{z})^{2}}\right), \\
g_{2}=\varphi_{1}^{\perp}\left(\partial g_{1}\right)=(0,0,0,0,0),
\end{gathered}
$$

and $\varphi_{2}$, determined by $f_{2}$ and $g_{2}$, is given by

$$
\varphi_{2}=\frac{1}{(1+z \bar{z})^{2}}\left(\begin{array}{ccccc}
z^{2} \bar{z}^{2} & 0 & -\sqrt{2} z^{2} \bar{z} & 0 & z^{2} \\
0 & 0 & 0 & 0 & 0 \\
-\sqrt{2} z \bar{z}^{2} & 0 & 2 z \bar{z} & 0 & -\sqrt{2} z \\
0 & 0 & 0 & 0 & 0 \\
\bar{z}^{2} & 0 & -\sqrt{2} \bar{z} & 0 & 1
\end{array}\right)
$$

$\varphi_{2}$ is an anti-holomorphic curve, which is isomorphic to the Veronese curve, in $\mathbf{C P}^{2}$.

Hence we obtain a harmonic sequence from $\varphi_{0}$ :

$$
0 \stackrel{\partial^{\prime}}{\longrightarrow} \varphi=\varphi_{0} \stackrel{\partial^{\prime}}{\longrightarrow} \varphi_{1} \stackrel{\partial^{\prime}}{\longrightarrow} \varphi_{2} \stackrel{\partial^{\prime}}{\longrightarrow} 0 .
$$

By a straightforward computation we obtain

$$
l_{0}=\frac{3}{(1+z \bar{z})^{2}}, \quad l_{1}=\frac{2}{(1+z \bar{z})^{2}}, \quad l_{2}=0 .
$$

It is very easy to see that $K\left(\varphi_{0}\right)=4 / 3, K\left(\varphi_{1}\right)=4 / 5, K\left(\varphi_{2}\right)=2$ and $t_{1}=3 / 2$.

It is well known that the rigidity of holomorphic curves in Grassmannians fails; so this example is a special harmonic sequence.

\section{Pinching Theorem for Curvature And Kähler Angle}

In this section we will discuss curvature pinching and Kähler angle pinching of non-degenerate pseudo-holomorphic spheres in complex Grassmann manifolds.

Let $\varphi=\varphi_{\alpha}: \mathbf{S}^{2} \rightarrow \mathbf{G}_{k_{\alpha}, n}$ be a pseudo-holomorphic curve with the nondegenerate associated harmonic sequence (5), and let $\alpha_{0}$ be the length of its associated harmonic sequence. Then from (28) we have

$$
\delta_{\alpha}=-\delta_{\alpha-2}+2 \delta_{\alpha-1}-\tau_{\alpha-1}-2
$$

for $\alpha=1, \cdots, \alpha_{0}$, and

$$
\tau_{\alpha}=\left(\delta_{\alpha}-\delta_{\alpha+1}\right)-\left(\delta_{\alpha-1}-\delta_{\alpha}\right)-2
$$

for $\alpha=0,1, \cdots, \alpha_{0}-1$. 
It is an immediate consequence of (41) and (42) that

$$
\delta_{\alpha}=(\alpha+1)\left(\delta_{0}-\alpha\right)-\sum_{\beta=0}^{\alpha-1}(\alpha-\beta) \tau_{\beta}
$$

for $\alpha=1, \cdots, \alpha_{0}$, and

$$
\tau_{0}+\cdots+\tau_{\alpha}=\left(\delta_{\alpha}-\delta_{\alpha+1}\right)+\delta_{0}-2(\alpha+1)
$$

for $\alpha=0,1, \cdots, \alpha_{0}-1$, where $\delta_{0}$ is the degree of the holomorphic map $\varphi_{0}$ in (5).

From (43) and (44) we have also

$$
\sum_{\alpha=0}^{\alpha_{0}-1}\left(\alpha_{0}-\alpha\right) \tau_{\alpha}=\left(\alpha_{0}+1\right)\left(\delta_{0}-\alpha_{0}\right)
$$

and

$$
\delta_{\alpha}=(\alpha+1)\left(\alpha_{0}-\alpha\right)+\frac{\alpha_{0}-\alpha}{\alpha_{0}+1} \sum_{\beta=0}^{\alpha-1}(\beta+1) \tau_{\beta}+\frac{\alpha+1}{\alpha_{0}+1} \sum_{\beta=\alpha}^{\alpha_{0}-1}\left(\alpha_{0}-\beta\right) \tau_{\beta} .
$$

Denoting $\tau=\min \left\{\tau_{0}, \cdots, \tau_{\alpha_{0}-1}\right\}$ ( $\geq 0$ ), we immediately obtain

$$
\delta_{0} \geq \alpha_{0}\left(1+\frac{1}{2} \tau\right), \quad \delta_{\alpha} \geq(\alpha+1)\left(\alpha_{0}-\alpha\right)\left(1+\frac{1}{2} \tau\right),
$$

and "=" holds if and only if $\tau_{0}=\cdots=\tau_{\alpha_{0}-1}$, where $\alpha=0,1, \cdots, \alpha_{0}-1$.

Obviously, $\varphi$ is a totally unramified non-degenerate pseudo-holomorphic minimal immersion, i.e., the harmonic sequence $\varphi_{0}, \cdots, \varphi_{\alpha_{0}}: \mathbf{S}^{2} \rightarrow \mathbf{G}_{k, n}$ is non-degenerate and totally unramified if and only if the degree $\delta_{0}$ of $\varphi_{0}$ is $\alpha_{0}$. For a totally unramified non-degenerate harmonic sequence $\varphi_{0}, \cdots, \varphi_{\alpha_{0}}: \mathbf{S}^{2} \rightarrow \mathbf{G}_{k, n}$ we have

$$
\delta_{\alpha}=(\alpha+1)\left(\alpha_{0}-\alpha\right) .
$$

At first, by using the Gauss-Bonnet theorem we have

Lemma 5.1. Suppose that the curvature $K_{\alpha}$ of $\varphi_{\alpha}$ satisfies either $K_{\alpha} \geq \frac{4}{\delta_{\alpha-1}+\delta_{\alpha}}$ or $K_{\alpha} \leq \frac{4}{\delta_{\alpha-1}+\delta_{\alpha}}$. Then $K_{\alpha}=\frac{4}{\delta_{\alpha-1}+\delta_{\alpha}}$.

Remark. In Lemma 5.1 we do not need to assume that $\varphi_{\alpha}$ is non-degenerate.

Theorem 5.2. Let $\varphi: \mathbf{S}^{2} \rightarrow \mathbf{G}_{k, n}$ be a pseudo-holomorphic curve with nondegenerate associated harmonic sequence, and suppose that $\varphi$ is the $\alpha$-th element $\varphi_{\alpha}$ of its non-degenerate associated harmonic sequence.

(i) If $K(\varphi) \geq \frac{4}{\left(\alpha_{0}+2 \alpha\left(\alpha_{0}-\alpha\right)\right)\left(1+\frac{1}{2} \tau\right)}$, then

$$
K(\varphi)=\frac{4}{\left(\alpha_{0}+2 \alpha\left(\alpha_{0}-\alpha\right)\right)\left(1+\frac{1}{2} \tau\right)}
$$

and $\tau_{\beta}=\tau$ for all $\beta$.

(ii) If $K(\varphi) \leq \frac{4}{\left(\alpha_{0}+2 \alpha\left(\alpha_{0}-\alpha\right)\right)\left(1+\frac{1}{2} \tau\right)}$ and if $\tau_{\beta}=\tau$ for all $\beta$, then

$$
K(\varphi)=\frac{4}{\left(\alpha_{0}+2 \alpha\left(\alpha_{0}-\alpha\right)\right)\left(1+\frac{1}{2} \tau\right)} .
$$


Proof. From (47) we see that

$$
\delta_{\alpha-1}+\delta_{\alpha} \geq\left(\alpha_{0}+2 \alpha\left(\alpha_{0}-\alpha\right)\right)\left(1+\frac{1}{2} \tau\right),
$$

with equality if and only if $\tau_{\beta}=\tau$ for all $\beta$. The result is now immediate from Lemma 5.1.

Remark. We have $t_{\alpha}=\frac{\alpha\left(\alpha_{0}-\alpha+1\right)}{(\alpha+1)\left(\alpha_{0}-\alpha\right)}$ under the assumption of Theorem 5.2. This shows that the Kähler angle $\theta_{\alpha}$ is independent of $\tau$.

The following is an immediate consequence of Theorem 5.2.

Corollary 5.3. Let $\varphi: \mathbf{S}^{2} \rightarrow \mathbf{G}_{k, n}$ be a holomorphic curve with non-degenerate associated harmonic sequence. Suppose $K(\varphi) \leq \frac{4}{\alpha_{0}\left(1+\frac{1}{2} \tau\right)}$ and $\tau_{\beta}=\tau$ for all $\beta$. Then $K(\varphi)=\frac{4}{\alpha_{0}\left(1+\frac{1}{2} \tau\right)}$.

Similarly, the following theorem is also an immediate consequence of Theorem 5.2 .

Corollary 5.4. Let $\varphi: \mathbf{S}^{2} \rightarrow \mathbf{G}_{k, n}$ be a holomorphic curve with non-degenerate associated harmonic sequence, and suppose $K(\varphi) \geq \frac{4}{\alpha_{0}\left(1+\frac{1}{2} \tau\right)}$. Then $K(\varphi)=$ $\frac{4}{\alpha_{0}\left(1+\frac{1}{2} \tau\right)}$, and $\tau_{\beta}=\tau$ for all $\beta$.

We now prove a pinching theorem for the Kähler angle. Let $\varphi: \mathbf{S}^{2} \rightarrow \mathbf{G}_{k, n}$ be a pseudo-holomorphic sphere and let $\varphi_{0}, \cdots, \varphi_{\alpha_{0}}$ be the associated harmonic sequence. We assume that $\varphi=\varphi_{\alpha}$.

Lemma 5.5 ([1]). If the Kähler angle $t_{\alpha}$ of $\varphi_{\alpha}$ satisfies either $t_{\alpha} \geq \frac{\delta_{\alpha-1}}{\delta_{\alpha}}$ or $t_{\alpha} \leq \frac{\delta_{\alpha-1}}{\delta_{\alpha}}$, then $t_{\alpha}=\frac{\delta_{\alpha-1}}{\delta_{\alpha}}$.

Lemma 5.6. Let $\varphi_{\alpha}$ be a pseudo-holomorphic curve with non-degenerate associated harmonic sequence. If $\tau_{\beta}=\tau$ for all $\beta$, and $t_{\alpha}$ satisfies either $t_{\alpha} \geq \frac{\alpha\left(\alpha_{0}-\alpha+1\right)}{(\alpha+1)\left(\alpha_{0}-\alpha\right)}$ or $t_{\alpha} \leq \frac{\alpha\left(\alpha_{0}-\alpha+1\right)}{(\alpha+1)\left(\alpha_{0}-\alpha\right)}$, then $t_{\alpha}=\frac{\alpha\left(\alpha_{0}-\alpha+1\right)}{(\alpha+1)\left(\alpha_{0}-\alpha\right)}$.

The proof of the above theorem is immediate from Lemma 5.5 and (47).

Using (46), we can also prove the following.

Theorem 5.7. Let $\varphi: \mathbf{S}^{2} \rightarrow \mathbf{G}_{k, n}$ be a pseudo-holomorphic curve with nondegenerate associated harmonic sequence, and suppose that $\varphi$ is the $\alpha$-th element $\varphi_{\alpha}$ of its non-degenerate associated harmonic sequence. If $t_{\alpha} \leq \frac{1}{2}$ (resp. $\left.t_{\alpha} \geq 2\right)$, then $t_{\alpha}=0$ (resp. $\left.t_{\alpha}=\infty\right)$, i.e., $\varphi$ is a holomorphic (resp. anti-holomorphic) curve.

Proof. When $\alpha \neq 0$ and $\alpha_{0}$, by (46) an immediate computation shows that

$$
\frac{1}{2}<\frac{\delta_{\alpha-1}}{\delta_{\alpha}}<2
$$

Hence, by Lemma 5.5, if $t_{\alpha} \leq \frac{1}{2}$ (resp. $t_{\alpha} \geq 2$ ), then $\alpha=0$ (resp. $\alpha=\alpha_{0}$ ), i.e., $\varphi$ is a holomorphic (resp. anti-holomorphic) curve. 
We believe that $\tau \neq 0$ for the non-degenerate harmonic sequence associated to the holomorphic curve of constant curvature, except for the Veronese sequence.

\section{REFERENCES}

[1] J. Bolton, G. R. Jensen, M. Rigoli, and L. M. Woodward, On conformal minimal immersions of $\mathbf{S}^{2}$ into $\mathbf{C P}^{n}$, Math. Ann., 279(1988), 599-620. MR 88m:53110

[2] E. Calabi, Isometric embedding of complex manifolds, Ann. Math. (2), 58(1953), 1-23. MR 15:160c

[3] S. S. Chern and J. G. Wolfson, Harmonic maps of the two-sphere into a complex Grassmann manifold, II, Ann. Math., 125(1987), 301-335. MR 88g:58038

[4] S. S. Chern and J. G. Wolfson, Minimal surfaces by moving frames, Amer. J. Math., 105(1983), 59-83. MR 84i:53056

[5] Q. Chi and Y. Zheng, Rigidity of pseudo-holomorphic curves of constant curvature in Grassmann manifolds, Trans. Amer. Math. Soc., 313(1989), 393-406. MR 90m:53072

[6] P. Griffiths and J. Harris, Principles of algebraic geometry, Pure and Applied Mathematics, London, New York: Wiley, 1978. MR 80b:14001

[7] X. X. Jiao, On harmonic maps of surfaces into complex Grassmannians, Chinese Ann. Math., 21A(1)(2000), 57-60. MR 2001b:53083

[8] H. B. Lawson, The Riemannian geometry of holomorphic curves, Proc. Conf. Holomorphic Mapping and Minimal Surfaces, Bol. Soc. Brasil. Mat., 2(1971), 45-62. MR 48:2957

[9] K. Uhlenbeck, Harmonic maps into Lie groups (classical solutions of the chiral model), J. Differential Geom., 30(1989), 1-50. MR 90g:58028

[10] J. G. Wolfson, Harmonic sequences and harmonic maps of surfaces into complex Grassmann manifolds, J. Differential Geom., 27(1988), 161-178. MR 89c:58031

[11] Y. B. Zheng, Quantization of curvature of harmonic two-spheres in Grassmann manifolds, Trans. Amer. Math. Soc., 316(1)(1989), 193-214. MR 90b:58055

[12] K. Yang, Complete and compact minimal surfaces, Kluwer Academic Publishers, 1989. MR 91h:53058

[13] K. Yang, Compact Riemann surfaces and algebraic curves, Series in Pure Mathematics, Vol. 10, World Scientific, 1988. MR 90e: 14023

[14] X. X. Jiao and J. G. Peng, A classification of holomorphic two-spheres with constant curvature in complex Grassmannians, Differential Geom. Appl., to appear.

Department of Mathematics, Graduate School, Chinese Academy of Sciences, Beijing 100039, CHINA

E-mail address: xxj@gscas.ac.cn

Department of Mathematics, Graduate School, Chinese Academy of Sciences, Beijing 100039, CHINA

E-mail address: pengck@gscas.ac.cn 